\title{
TWA - Ticket Locks Augmented with a Waiting Array
}

\author{
Dave Dice (1) \\ Oracle Labs \\ first.last@oracle.com
}

\author{
Alex Kogan (1) \\ Oracle Labs \\ first.last@oracle.com
}

\begin{abstract}
The classic ticket lock $[22,35,39]$ consists of ticket and grant fields. Arriving threads atomically fetch-and-increment ticket to obtain an assigned ticket value, and then wait for grant to become equal to that value, at which point the thread holds the lock. The corresponding unlock operation simply increments grant. This simple design has short code paths and fast handover (transfer of ownership) under light contention, but may suffer degraded scalability under high contention when multiple threads busy wait on the grant field - so-called global spinning.

We propose a variation on ticket locks where long-term waiting threads - those with an assigned ticket value far larger than grant - wait on locations in a waiting array instead of busy waiting on the grant field. The single waiting array is shared among all locks. Short-term waiting is accomplished in the usual manner on the grant field. The resulting algorithm, TWA, improves on ticket locks by limiting the number of threads spinning on the grant field at any given time, reducing the number of remote caches requiring invalidation from the store that releases the lock. In turn, this accelerates handover, and since the lock is held throughout the handover operation, scalability improves. Under light or no contention, TWA yields performance comparable to the classic ticket lock. Under high contention, TWA is substantially more scalable than the classic ticket lock, and provides performance on par or beyond that of scalable queue-based locks such as MCS [35] by avoiding the complexity and extra accesses incurred by the MCS handover operation while also obviating the need for maintaining queue elements.

We provide an empirical evaluation, comparing TWA against ticket locks and MCS for various user-space applications, and within the Linux kernel.
\end{abstract}

CCS Concepts - Software and its engineering $\rightarrow M u l-$ tithreading; Mutual exclusion; Concurrency control; Process synchronization;

Keywords Locks, Mutexes, Mutual Exclusion, Synchronization, Concurrency Control

\section{Introduction}

The classic ticket lock is compact and has a very simple design. The acquisition path requires only one atomic operation - a fetch-and-add to increment the ticket - and the unlock path requires no atomics. On Intel systems, fetchand-add is implemented via the LOCK: XADD instruction so the doorway phase [28] is wait-free. Under light or no contention, the handover latency, defined as the time between the call to unlock and the time a successor is enabled to enter the critical section, is low. Handover time impacts the scalability as the lock is held throughout handover, increasing the effective length of the critical section [3,21]. Typical implementations use 32-bit integers for the ticket and grant variable. Rollover is not a concern as long as the number of concurrently waiting threads on a given lock never exceeds $2^{32}-1^{1}$. A ticket lock is in unlocked state when ticket and grant are equal. Otherwise the lock is held, and the number of waiters is given by ticket - grant - 1. Ignoring numeric rollover, grant always lags or is equal to ticket. The increment operation in unlock either passes ownership to the immediate successor, if any, and otherwise sets the state to unlocked.

Ticket locks suffer, however, from a key scalability impediment. All threads waiting for a particular lock will busy wait on that lock's grant field. An unlock operation, when it increments grant, invalidates the cache line underlying grant for all remote caches where waiting threads are scheduled. In turn, this negatively impacts scalability by retarding the handover step. Ticket locks use global spinning, as all waiting threads monitor the central lock-specific grant variable.

In Figure-1 we show the impact of readers on a single writer. We refer to the number of participating caches as the invalidation diameter [19]. The Invalidation Diameter benchmark spawns $T$ concurrent threads, with $T$ shown on the $\mathrm{X}$-axis. A single writer thread loops, using an atomic fetch-and-add primitive to update a shared location. The other $T-1$ threads are readers. They loop, fetching the value of that location. The shared variable is sequestered to avoid false sharing and is the sole occupant of its underlying cache sector. We present the throughput rate of the writer on the Y-axis. As we increase the number of concurrent readers, the writer's progress is slowed. This scenario models the situation in ticket locks where multiple waiting threads monitor the grant field, which is updated by the current owner during handover. The benchmark reports the writer's throughput at the end of a 10 second measurement interval. The data exhibited high variance due to the NUMA placement vagaries of the threads and the home node of the variable. As such, for each data point show, we took the median of 100 individual runs, reflecting a realistic set of samples. The system-under-test is described in detail in $\S 4$.

\footnotetext{
${ }^{1}$ Developers assume this constraint is always satisfied - having more that $2^{32}-1$ waiting threads is not considered a practical concern.
} 
The MCS lock [35] is the usual alternative to ticket locks, performing better under high contention, but also having a more complex path and often lagging behind ticket locks under no or light contention. In MCS, arriving threads use an atomic operation to append an element, commonly called a "queue node", to the tail of a linked list of waiting threads, and then busy wait on a field within that element, avoiding global spinning. The list forms a queue of waiting threads. The lock's tail variable is explicit and the head - the current owner - is implicit. When the owner releases the lock it reclaims the element it originally enqueued and sets the flag in the next element, passing ownership. To convey ownership, the MCS unlock operator must identify the successor, if any, and then store to the location where the successor busy waits. The handover path is longer than that of ticket locks and accesses more distinct shared locations. MCS uses so-called local waiting where at most one thread is waiting on a given location at any one time. As such, an unlock operation will normally need to invalidate just one location the flag where the successor busy waits. Under contention, the unlock operator must fetch the address of the successor node from its own element, and then store into the flag in the successor's element, accessing two distinct cache lines, and incurring a dependent memory access to reach the successor. In the case of no contention, the unlock operator must use an atomic compare-and-swap operator to detach the owner's element.

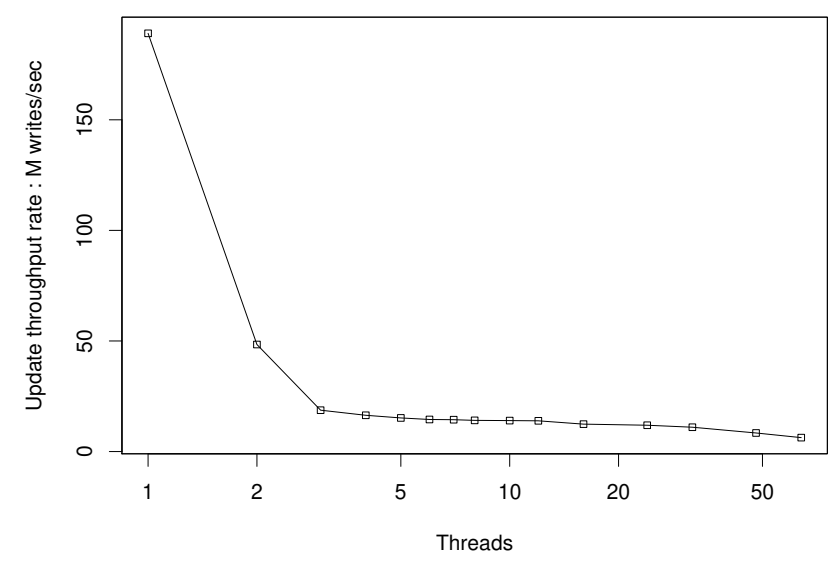

Figure 1. Invalidation Diameter

One MCS queue node instance is required for each lock a thread currently holds, and an additional queue node is required while a thread is waiting on a lock. Queue nodes can not be shared concurrently and can appear on at most one queue - be associated with at most one lock - at a given time. The standard POSIX pthread_mutex_lock and pthread_mutex_unlock operators do not require scoped or lexically balanced locking. As such, queue nodes can not be allocated on stack. Instead, MCS implementations that expose a standard POSIX interface will typically allocate elements from thread-local free lists, populated on demand 2

The standard POSIX interface does not provide any means to pass information from a lock operation to the corresponding unlock operator. As such, the address of the MCS queue node inserted by the owner thread is usually recorded in the lock instance so it can be conveyed to the subsequent unlock operation to identify the successor, if any. Accesses to the field that records the owner's queue node address may themselves generate additional coherence traffic, although some implementations may avoid such accesses to shared fields by storing the queue node address in a thread-local associative structure that maps lock addresses to the owner's queue node address.

Ticket locks and TWA require no indirection or dependent accesses in the unlock path and also avoid the need for queue elements and the management thereof. The queue of waiting threads is implicit in ticket locks and TWA, and explicit in MCS. MCS, ticket locks and TWA all provide strict FIFO admission order.

Ticket locks are usually a better choice under light or no contention, while MCS locks are more suitable under heavy contention [6, 8]. By employing a waiting array for long-term waiting, TWA achieves the best of the two worlds, as demonstrated by our empirical evaluation with multiple user-space applications and within the Linux kernel ${ }^{3}$.

We note in passing that under conditions of intermittent or no contention when no successor is visible, MCS may require an atomic compare-and-swap operation to detach the onwer's queue node from the MCS tail pointer. (When waiting threads are visible in the MCS chain, the MCS unlock operator accomplish succession with just a store into the next thread's queue node structure). In contrast, ticket locks never require an atomic read-modify-write instruction in the unlock path. We do not believe, however, that the atomic instruction accounts for the difference in performance between MCS and ticket locks at low contention. For instance if we replace the load-increment-store sequence found in the unlock path of the ticket lock algorithm with an atomic fetchand-add, we observe no appreciable change in performance. Broadly, on modern processors, we find that coherence traffic dictates performance, and that an atomic compare-and-swap is the perforamnce equivalent of a simple store.

\footnotetext{
${ }^{2}$ Threads might also malloc and free queue nodes as needed, but most malloc allocators are not sufficiently scalable. Also, many malloc implementations themselves make use of POSIX locks, resulting in reentry and recursion if a lock implementation were to try to call malloc which in turn would need to acquire a lock. We note that the MCS "K42" variant $[32,40]$ allows queue nodes to be allocated on stack - they are required only while a thread waits - but at the cost of a longer path with more accesses to shared locations.

${ }^{3}$ The Linux kernel switched from ticket locks to MCS-based locks in 2014 [14].
} 


\section{The TWA Algorithm}

TWA builds directly on ticket locks. We add a new waiting array for long-term waiting. The array is shared amongst all threads and TWA locks in an address space. Arriving threads use an atomic fetch-and-increment instruction to advance the ticket value, yielding the lock request's assigned ticket value, and then fetch grant. If the difference is 0 then we have uncontended acquisition and the thread may enter the critical section immediately. (This case is sometimes referred to as the lock acquisition fast-path). Otherwise TWA compares the difference to the LongTermThreshold parameter. If the difference exceeds LongTermThreshold then the thread enters the long-term waiting phase. Otherwise control proceeds to the short-term waiting phase, which is identical to that of normal ticket locks; the waiting thread simply waits for grant to become equal to the ticket value assigned to the thread. While LongTermThreshold is a tunable parameter in our implementation, we found a value of 1 to be suitable for all environments, ensuring that only the immediate successor waits in short-term mode. All data reported below uses a value of 1 .

A thread entering the long-term waiting phase first hashes its assigned ticket value to form an index into the waiting array. Using this index, it fetches the value from the array and then rechecks the value of grant ${ }^{4}$. If the observed grant value changed, the thread rechecks the difference between that new value and its assigned ticket value, and decides once again on short-term versus long-term waiting. If grant was unchanged, the thread then busy waits for the waiting array value to change, at which point it reevaluates grant. When grant is found to be sufficiently near the assigned ticket value, the thread reverts to normal short-term waiting. The values found in the waiting array have no particular meaning, except to conservatively indicate that a grant value that maps to that index has changed, and rechecking of grant is required for waiters on that index. As rollover is a concern in the waiting array, we use 64-bit integers, so in practice, rollover never occurs.

The TWA unlock operator increments grant as usual from $U$ to $U+1$ and then uses an atomic operator to increment the location in the waiting array that corresponds to threads waiting on ticket value $U+1+$ LongTermThreshold, notifying long-term threads, if any, that they should recheck grant. An atomic operation is necessary as the location may be subject to hash collisions. We observe that this change increases the path length in the unlock operator, but crucially the store that effects handover, which is accomplished

\footnotetext{
${ }^{4}$ The recheck step is needed to avoid races between lock and unlock operations. Specifically, in the window between the load of grant and the recheck, the owner might have released the lock. Absent the recheck, the algorithm would be vulnerable to lost wakeups. Similar recheck idioms appear in other constructs, such as the Linux kernel futex mechanism, where waiting threads check a condition, prepare for long-term waiting, and then recheck the condition before commiting to long-term waiting.
}

by a non-atomic increment of grant, happens first. Given a LongTermThreshold value of 1 , we expect at most one thread, the immediate successor, to be waiting on grant. Updating the waiting array occurs after handover and outside the critical section.

All our experiments use a waiting array with 4096 elements, although ideally, we believe the waiting array should be sized as a function of the number of CPUs in the system. (A similar approach is used to size the futex hash table array in the Linux kernel ${ }^{5}$.) Hash collisions in the table are benign, at worst causing unnecessary rechecking of the grant field. Specifically, collisions are a performance and qualityof-implementation concern that does not impact correctness. A larger waiting array table will reduce the collisions rate but might increase cache pressure. We note that the odds of inter-lock collision are equivalent to those given by the "Birthday Paradox" [43]. Our hash function is cache-aware and intentionally designed to map adjacent ticket values to different 128-byte cache sectors underlying the waiting array, to reduce false sharing among long-term waiters. We multiply the ticket value by 127 and then EXCLUSIVE-OR that result with the address of the lock, and then mask with 4096 - 1 to form an index into the waiting array. We selected a small prime $P=127$ to provide the equidistribution properties of a Weyl sequence [33] and also to thwart the automatic stride-based hardware prefetch mechanism which can artifically induce false sharing. Multiplication by 127 is easily strength-reduced to a shift and subtract. We include the lock address into our deterministic hash to avoid the situation where two locks might operate in an entrained fashion, with ticket and grant values moving in near unison, and thus suffer from excessive inter-lock collisions. A given lock address and ticket value pair always hashes to the same index. The hash computed in the unlock operator must target the same index as the corresponding hash in the long-term waiting path. We also note that near collisions can result in false sharing, when two accesses map to distinct words in the same cache sector.

TWA leaves the structure of the ticket lock unchanged, allowing for easy adoption. As the instance size remains the same, the only additional space cost for TWA is the waiting array, which is shared over all locks, reflecting a one-time space cost.

The TWA fast-path for acquisition remains unchanged relative to ticket locks. The unlock path adds an increment of the waiting array, to notify any long-term waiters sufficiently "near" the front of the queue that they should transition from long-term to short-term waiting. We note that TWA doesn't reduce overall coherence traffic, but does act to reduce coherence traffic in the critical handover path, constraining the invalidation diameter of the store in unlock that accomplishes handover. TWA thus captures the

\footnotetext{
${ }^{5}$ https://blog.stgolabs.net/2014/01/futexes-and-hash-table-collisions.html
} 
desirable performance aspects of both MCS locks and ticket locks.

Listing-1 depicts a pseudo-code implementation of the TWA algorithm. Lines 7 through 16 reflect the classic ticket lock algorithm and lines 20 through 71 show TWA. TWA extends the existing ticket lock algorithm by adding lines 41 through 57 for long-term waiting, and line 71 to notify long-term waiters to shift to classic short-term waiting.

\subsection{Example Scenario - TWA in Action}

(1) Initially the lock is in unlocked state with Ticket and Grant both 0 .

2 Thread $T 1$ arrives at Listing-1 line 34 attempting to acquire the lock. $T 1$ increments Ticket from 0 to 1 , and the atomic FetchAdd operator returns the original value of 0 into the local variable $t x$, which holds the assigned ticket value for the locking request. At line $36 \mathrm{T1}$ then fetches Grant observing a value of 0 . Since $t x$ equals that fetched value, we have uncontended lock acquisition. T1 now holds the lock and can enter the the critical section immediately, without waiting, via the fast path at line 39.

(3) Thread T2 now arrives and tries to acquire the lock. The FetchAdd operator advances Ticket from 1 to 2 and returns 1 , the assigned ticket, into $t x$ at line 35. T2 fetches Grant and notes that tx differs from that value by 1 . The $\mathrm{dx}$ variable holds that computed difference, which reflects the number of threads between the requester and the head of the logical queue, which is the owner. T2 has encountered contention and must wait. The difference is only 1 , and $T 2$ will be the immediate successor, so $T 2$ proceeds to line 60 for short-term waiting similar to that used in classic ticket locks shown at line 10. T2 waits for the Grant field to become 1 .

(4) Thread T3 arrives and advances Ticket from 2 to 3, with the FetchAdd operator returning 2 as the assigned ticket. The difference between that value (2) and the value of Grant $(0)$ fetched at line 64 exceeds the LongTermThreshold (1), so T3 enters the path for long-term waiting at line 49. T3 hashes its observed ticket value of 2 into an index at, say 100, in the long-term waiting array and then fetches from WaitArray[at] observing $U$. To recover from potential races with threads in the unlock path, T3 rechecks that the Grant variable remains unchanged (0) at line 49 and that the thread should continue with long-term waiting. Thread T3 busy waits at lines 52-53 on the WaitArray value.

(5) Thread T4 arrives, advances Ticket from 3 to 4, obtaining a value in its tx variable of 3 . Similar to T3, T4 enters the long-term. T4 hashes its assigned ticket value of 3 yielding an index of, say, 207, and fetches Wai tArray [207] observing $V$. T4 then busy waits, waiting for WaitArray[207] to change from $V$ to any other value.
6 Thread T1 now releases the lock, calling TWARelease at line 63. $T 1$ increments Grant from 0 to 1 at line 67, passing ownership to $T 2$ and sets local variable $k$ to the new value (1).

(7) Thread T2 waiting at lines 60-61 notices that Grant changed to match its $t x$ value. T2 is now the owner and may enter the critical section.

8 Thread T1, still in TWARelease at line 71 then hashes $k+$ LongTermThreshold (the sum is 2) to yield index 100 and then increments WaitArray[100] from $U$ to $U+1$.

9 Thread T3 waiting at lines 52-53 observes that change, rechecks Grant, sees that it is close to being granted ownership, exits the long-term waiting loop and switches to classic short-term waiting at lines 60-61. T1 has promoted T3 from long-term to short-term waiting in anticipation of the next unlock operation, to eventually be performed by $T 2$.

10 Thread T1 now exits the TWARelease operator.

(11) Thread T2 is the current owner, thread T3 is waiting in short-term mode, and thread T4 is waiting in long-term mode.

\section{Related Work}

Mellor-Crummey and Scott [35] proposed ticket locks with proportional backoff. Waiting threads compare the value of their ticket against the grant field. The difference reflects the number of intervening threads waiting. That value is then multiplied by some tunable constant, and the thread delays for that period before rechecking grant. The intention is to reduce futile polling that might induce unnecessary coherence traffic. The constant is platform- and load-dependent, and requires tuning. In addition, while the approach decreases the futile polling rate on grant, and may be used in conjunction with polite waiting techniques [19], it does not decrease the invalidation diameter. TWA and ticket locks with proportional backoff both makes a distinction among waiting threads based on their relative position in the queue.

Partitioned Ticket Locks [18] augment each ticket lock with a constant-length private array of grant fields, allowing for semi-local waiting. Critically, the array is not shared between locks, and to avoid false sharing within the array, the memory footprint of each lock instance is significantly increased. Ticket Lock "AWN" [37] also uses per-lock array for semi-local waiting. Anderson's array-based queueing lock $[4,5]$ is also based on ticket locks. It employs a waiting array for each lock instance, sized to ensure there is at least one array element for each potentially waiting thread, yielding a potentially large footprint. The maximum number of participating threads must be known in advance when initializing the array. Such dynamic sizing also makes static allocation of Anderson's locks more difficult than would be the case for a lock with a fixed size, such as TWA. 


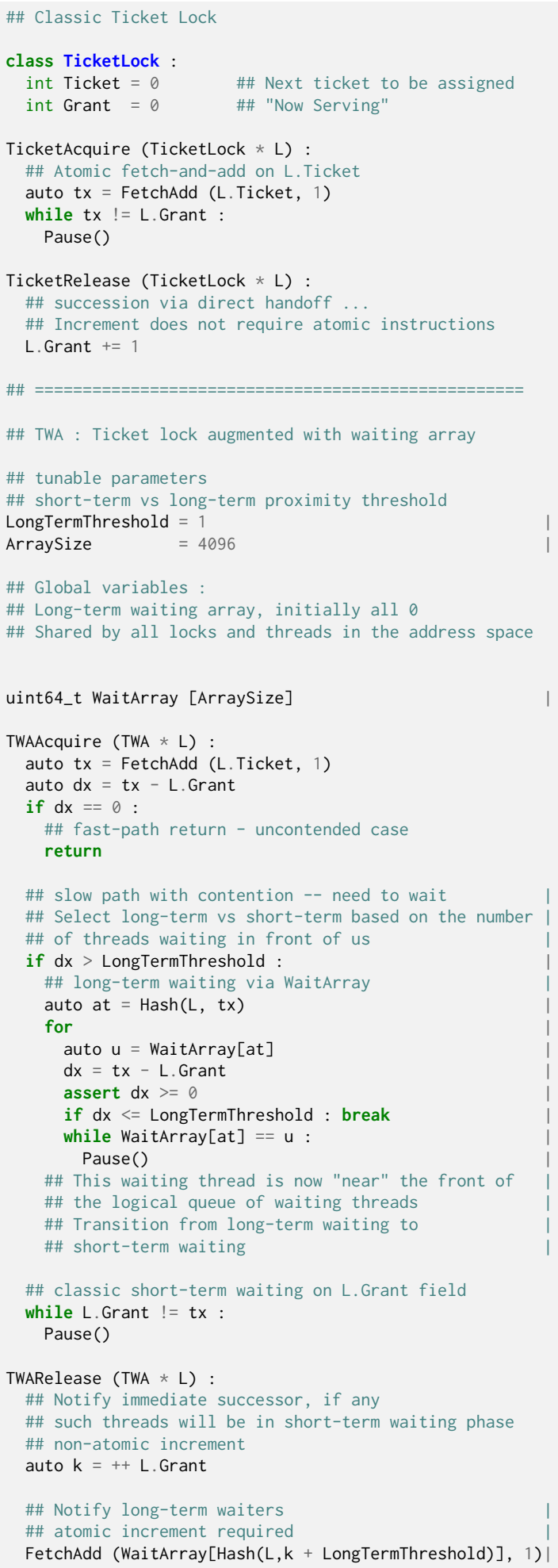

\section{Listing 1. Simplified Python-like Implementation of TWA}

Fu et al. [24, 29] describe a mutual exclusion scheme that avoids global spinning, but the technique has long paths and accesses large numbers of shared variables.
Various authors [6, 26] have suggested switching adaptively between MCS and ticket locks depending on the contention level. While workable, this adds considerable algorithmic complexity, particularly for the changeover phase, and requires tuning. Lim and Agarwal [30] suggested a more general framework for switching locks at runtime.

\section{Empirical Evaluation}

Unless otherwise noted, all data was collected on an Oracle X5-2 system. The system has 2 sockets, each populated with an Intel Xeon E5-2699 v3 CPU running at 2.30GHz. Each socket has 18 cores, and each core is 2-way hyperthreaded, yielding 72 logical CPUs in total. The system was running Ubuntu 18.04 with a stock Linux version 4.15 kernel, and all software was compiled using the provided GCC version 7.3 toolchain at optimization level “-O3”. 64-bit C or C++ code was used for all experiments. Factory-provided system defaults were used in all cases, and Turbo mode [42] was left enabled. In all cases default free-range unbound threads were used. SPARC and x 86 both provide a strong TSO memory model [41]. The atomic fetch-and-add primitives, LOCK: XADD on x86 and a LD; ADD; CAS loop on SPARC, have fence semantics. TWA is trivial to implement in $\mathrm{C}++$ with std: : atomic $<>$ primitives.

We implemented all user-mode locks within LD_PRELOAD interposition libraries that expose the standard POSIX pthread_ mutex_t programming interface using the framework from [20]. This allows us to change lock implementations by varying the LD_PRELOAD environment variable and without modifying the application code that uses locks. The $\mathrm{C}++$ std: : mutex construct maps directly to pthread_mutex primitives, so interposition works for both $\mathrm{C}$ and $\mathrm{C}++$ code. All busy-wait loops used the Intel PAUSE instruction for polite waiting.

We use a 128 byte sector size on Intel processors for alignment to avoid false sharing. The unit of coherence is 64 bytes throughout the cache hierarchy, but 128 bytes is required because of the adjacent cache line prefetch facility where pairs of lines are automatically fetched together.

\subsection{Sensitivity to Inter-Lock Interference}

As the waiting array is shared over all locks and threads within an address space, one potential concern is collisions that might arise when multiple threads are using a large set of locks. Near collisions are also of concern as they can cause false sharing within the array. To determine TWA's performance sensitity to such effects, we implemented a benchmark program that spawns 64 concurrent threads. Each thread loops as follows : randomly pick a lock from a pool of such locks; acquire that lock; advance a thread-local pseudorandom number generator 50 steps; release the lock; and finally advance that random number generator 100 steps. At the end of a 10 second measurement interval we report the 
number of lock acquistions. Each data point is the median of 7 distinct runs. We report the results in Figure- 2 where $\mathrm{X}$-axis reflects the number of locks in the pool (varying through powers-of-two between 1 and 8192) and the Y-axis is the number of acquisitions completed by TWA divided by the number completed by a specialized version of TWA where each lock instance has a private array of 4096 elements. This fraction reflects the performance drop attributable to interlock conflicts and near conflicts in the shared array, where the modified form of TWA can be seen as an idealized form that has a large per-instance footprint but which is immune to inter-lock conflicts. The worst-case penalty arising from inter-thread interference (the lowest fraction value) is always under $8 \%$.

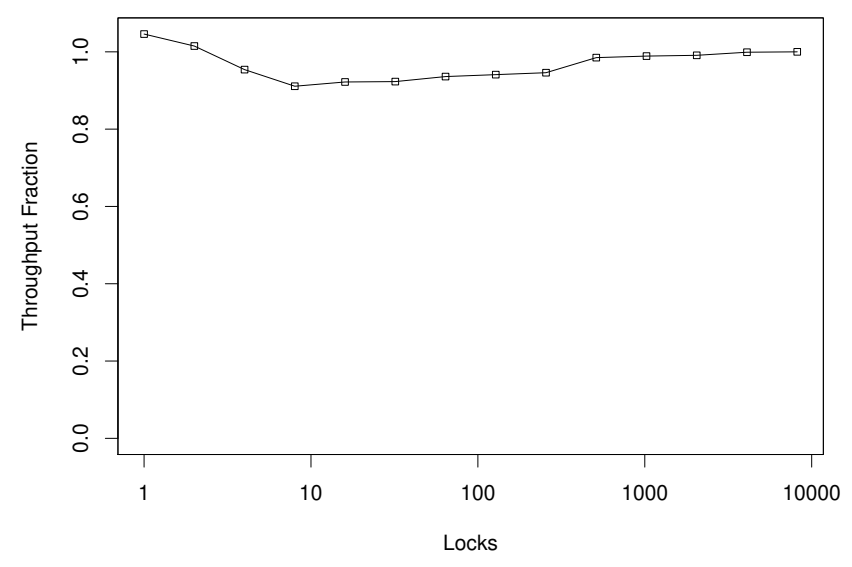

Figure 2. Inter-Lock Interference

\subsection{MutexBench}

The MutexBench benchmark spawns $T$ concurrent threads. Each thread loops as follows: acquire a central lock L; execute a critical section; release L; execute a non-critical section. At the end of a 10 second measurement interval the benchmark reports the total number of aggregate iterations completed by all the threads. We show the median of 5 independent runs in Figure-3. The critical section advances a C++ std: : mt 19937 pseudo-random generator (PRNG) 4 steps. The non-critical section uses that same PRNG to compute a value distributed uniformly in $[0,200)$ and then advances the PRNG that many steps. For clarity and to convey the maximum amount of information to allow a comparision the algorithms, the $X$-axis is offset to the minimum score and the $Y$-axis is logarithmic.

As seen in the figure, ticket locks performs the best up to 6 threads, with TWA lagging slightly behind. As we further increase the thread count, however, ticket locks fail to scale. MCS provides stable asymptotic performance that surpasses ticket locks at 24 threads. TWA manages to always outperform MCS, freeing the developer from making a choice between MCS locks and ticket locks.

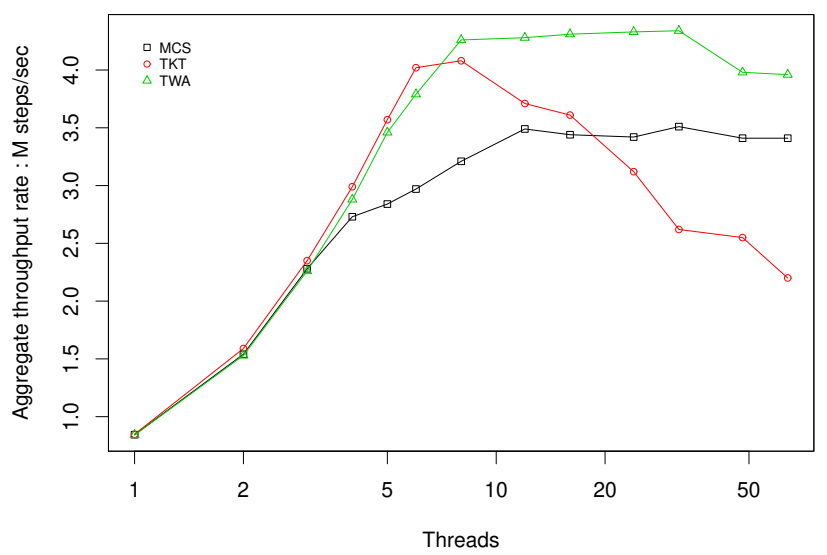

Figure 3. MutexBench

To show that our approach is general and portable, we next report MutexBench results on a Sun/Oracle T7-2 [15]. The T7-2 has 2 sockets, each socket populated by an M7 SPARC CPU running at $4.13 \mathrm{GHz}$ with 32 cores. Each core has 8 logical CPUs sharing 2 pipelines. The system has 512 logical CPUs and was running Solaris 11. 64-bit SPARC does not directly support atomic fetch-and-add or swap operations these are emulated by means of a 64-bit compare-and-swap operator (CASX). The system uses MOESI cache coherency instead of the MESIF [25] found in modern Intel-branded processors, allowing more graceful handling of write sharing. The graph in Figure-4 has the same shape as found in Figure3 . The abrupt performance drop experienced by all locks starting at 256 threads is caused by competition for pipeline resources.

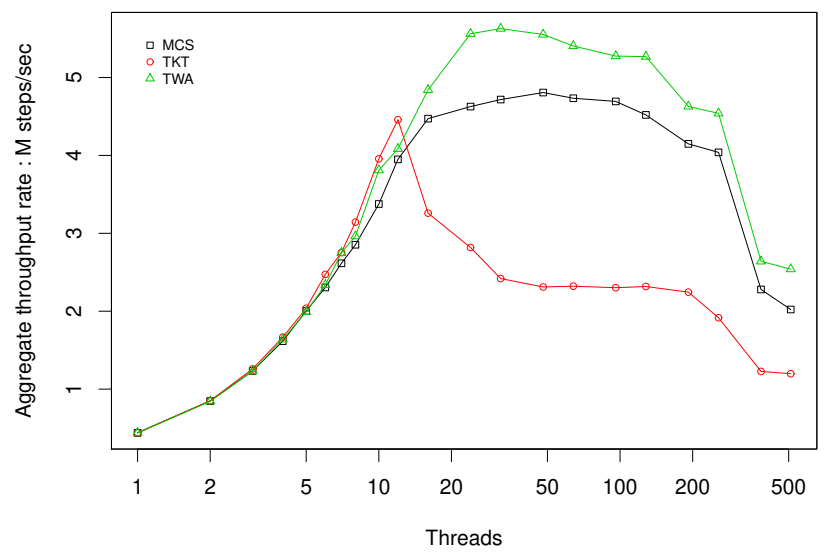

Figure 4. MutexBench on Solaris/SPARC 64-bit

\section{3 throw}

The "throw" benchmark launches $T$ threads, each of which loop, executing the following line of $\mathrm{C}++$ code:

try $\{$ throw 20 ; $\}$ catch (int e) \{\} . 
Naively, this construct would be expected to scale linearly, but the $\mathrm{C}++$ runtime implementation acquires mutexes that protect the list of dynamically loaded modules and their exception tables ${ }^{6}$. The problem is long-standing and has proven difficult to fix given the concern that some applications might have come to depend on the serialization [1]. At the end of a 10 second measurement interval the benchmark reports the aggregate number of loops executed by all threads. There is no non-critical section in this benchmark; throw-catch operations are performed back-to-back with no intervening delay. In Figure- 5 we observe that performance drops significantly between 1 and 2 threads. There is little or no benefit from multiple threads, given that execution is largely serialized, but coherent communication costs are incurred. As we increase beyond two threads performance improves slightly, but never exceeds that observed at one thread. Beyond 2 threads, the shape of the graph recapitulates that seen in MutexBench.

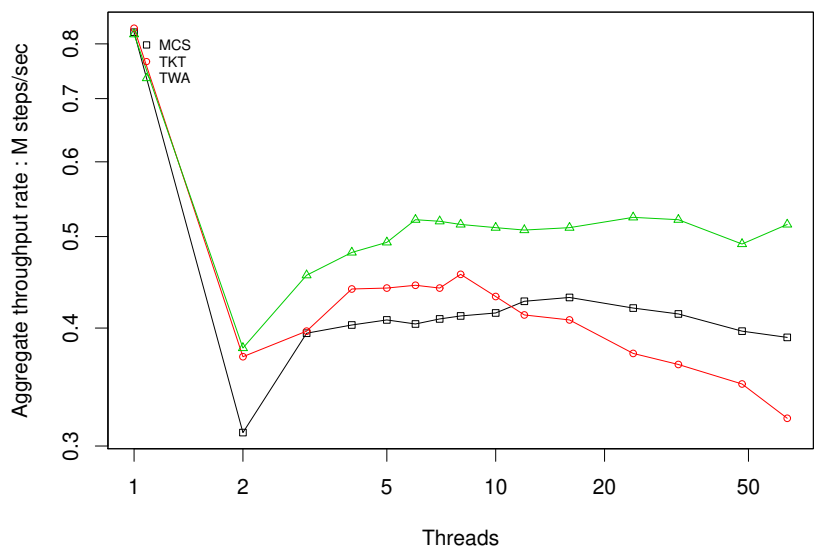

Figure 5. throw

\subsection{Random Replacement Cache}

The "Random Replacement Cache" benchmark creates a keyvalue cache with a random replacement policy ${ }^{7}$. All cache operations are protected with a central lock. Both the keys and values are 32-bit integers and we set values equal to a hash of the key. The cache is configured with a capacity limit of 10000 elements. The benchmark launches the specified number of concurrent threads, each of which loops, accessing the cache, and then executing a delay. At the end of a 10 second measurement interval the benchmark reports the aggregate throughput rate. We plot the median of 5 runs

\footnotetext{
${ }^{6}$ The $\mathrm{C}++$ runtime libraries have specialized binding conventions to invoke locking operators, which are not amenable to normal LD_PRELOAD interposition. We instead intercepted the locking calls via the _rtld_global facility.

${ }^{7}$ We use the cache from https://github.com/ceph/ceph/blob/master/src/ common/random_cache.hpp in conjunction with a test harness of our own making
}

for each data point in Figure-6. To emulate locality and key reuse, each thread has a private keyset of 10 recently used keys. We pre-populate the keyset with random keys before the measurement interval, using selection with replacement. With probability $P=0.9$ a thread picks a random index in its keyset and then uses the corresponding key for its access. We use thread-local C++ std: : mt 19937 pseudo-random number generators with a uniform distribution. Otherwise, the thread generates a new random key in the range $[0,50000)$, installs that key into a random slot in the keyset, and then proceeds to access the cache with that key. The inter-access delay operation picks a random number in the range $[0,200)$ and then steps the thread-local random number generator that many times.

The cache implementation makes frequent use of mallocfree operations. The default malloc allocator fails to fully scale in this environment and attenuates the benefit conferred by improved locks, so we instead used the index-aware allocator from [2]. This allocator uses its own built-in synchronization primitives instead of pthread operators, so LD_PRELOAD interposition on the pthread mutex primitives has no influence on malloc performance.

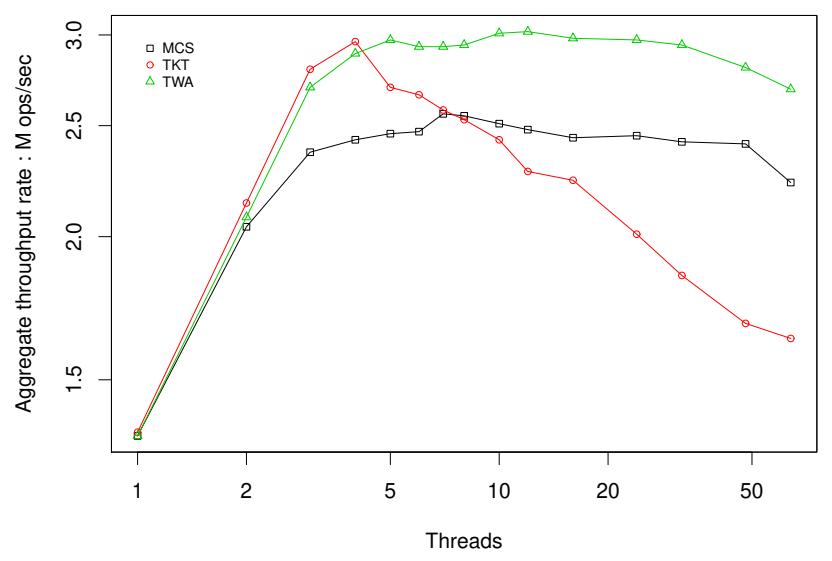

Figure 6. Random Replacement Cache

\section{5 libslock stress_latency}

Figure-7 shows the performance of the "stress latency" benchmark from $[17]^{8}$. The benchmark spawns the specified number of threads, which all run concurrently during a 10 second measurement interval. Each thread iterates as follows: acquire a central lock; execute 200 loops of a delay loop; release the lock; execute 5000 iterations of the same delay loop. The benchmark reports the total number of iterations of the outer loop.

\footnotetext{
${ }^{8}$ We use the following command line: ./stress_latency -1 1 -d 10000 -a 200 -n threads -w 1 -c 1 -p 5000.
} 


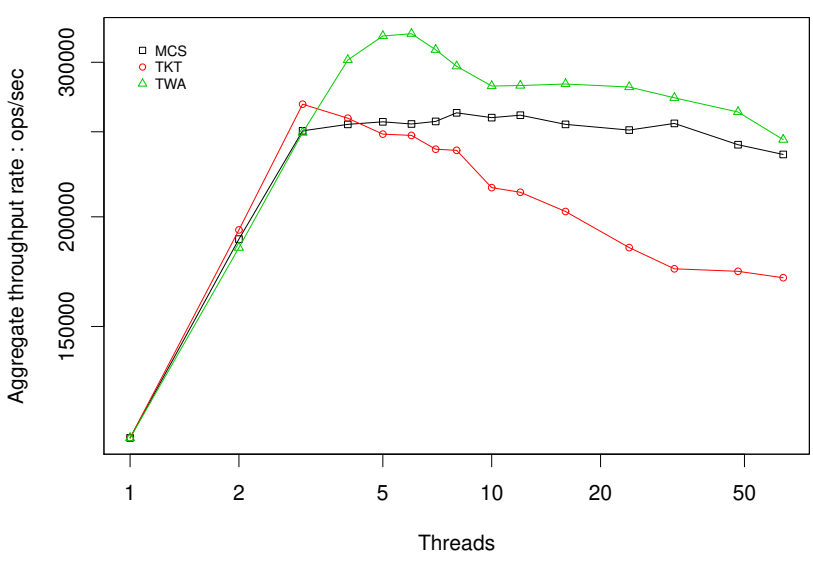

Figure 7. libslock stress_latency

\subsection{LevelDB readrandom}

In Figure-8 we used the "readrandom" benchmark in LevelDB version 1.20 database ${ }^{9}$ varying the number of threads and reporting throughput from the median of 5 runs of 50 second each. Each thread loops, generating random keys and then trying to read the associated value from the database. We first populated a database ${ }^{10}$ and then collected data ${ }^{11}$. We made a slight modification to the db_bench benchmarking harness to allow runs with a fixed duration that reported aggregate throughput. Ticket locks exhibit a very slight advantage over MCS and TWA at low threads count after which ticket locks fade and TWA matches or exceeds the performance of MCS. LevelDB uses coarse-grained locking, protecting the database with a single central mutex: DBImpl: : Mutex. Profiling indicates contention on that lock via leveldb: :DBImpl: :Get().

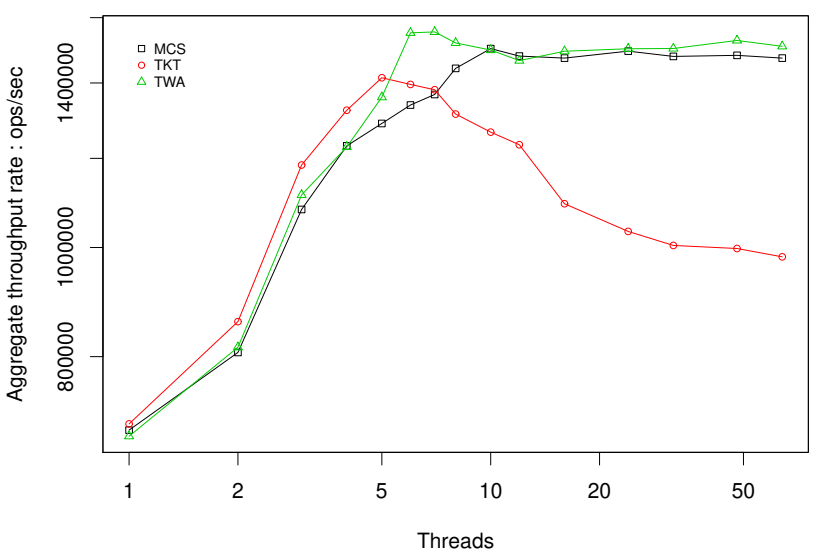

Figure 8. LevelDB readrandom

\footnotetext{
${ }^{9}$ leveldb.org

${ }^{10} \mathrm{db}$-bench - -threads $=1$ - -benchmarks $=$ fillseq $--\mathrm{db}=/ \mathrm{tmp} / \mathrm{db} /$

${ }^{11} \mathrm{db}$ _bench - -threads $=$ threads - -benchmarks=readrandom

--use_existing_db=1 - $-\mathrm{db}=/ \mathrm{tmp} / \mathrm{db} /-$-duration $=50$
}

\subsection{LevelDB readwhilewriting}

The LevelDB "readwhilewriting" benchmark in Figure-9 spawns $T-1$ random readers (identical to the "readrandom" threads) and a single writer thread which writes to randomly selected keys ${ }^{12}$. The benchmark reports the aggregate throughput completed in a 50 second measurement interval. Each data point is taken as median of 5 distinct runs. The same lock in "readrandom" is the source of contention in this benchmark.

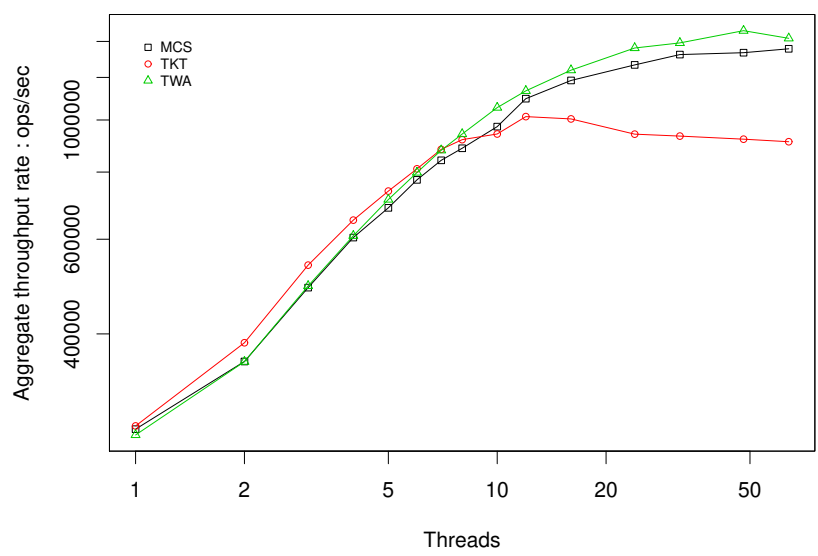

Figure 9. LevelDB readwhilewriting

\subsection{RocksDB readwhilewriting}

We next present results in Figure-10 from the RocksDB ${ }^{13}$ version 5.14.2 database running their variant of the "readwhitewriting" benchmark. The benchmark is similar to the form found in LevelDB, above, but the underlying database allows more concurrency and avoids the use of a single central lock. We intentionally use a command-line configured to stress the locks that protect the sharded LRU cache, causing contention in LRUShard: : lookup() ${ }^{14}$.

\subsection{Linux kernel locktorture}

We ported TWA into the Linux kernel environment and evaluated its performance with the locktorture benchmark ${ }^{15}$. Locktorture is distributed as a part of the Linux kernel. It is implemented as a loadable kernel module, and according to its documentation, "runs torture tests on core kernel locking primitives", including qspinlock, the kernel spin lock. It creates a given number of threads that repeatedly acquire and release the lock, with occasional short delays (citing the comment in the source code, "to emulate likely code")

\footnotetext{
${ }^{12} \mathrm{db}$ _bench --benchmarks=readwhilewriting - -threads=threads -cache_size $=50000--$ num $=100000--$ duration $=50$

${ }^{13}$ rocksdb.org

${ }^{14} \mathrm{db} \_$bench -- duration $=200-$-threads $=$ threads

-- benchmarks $=$ readwhilewriting -- compression $\_$type $=$none

--mmap_read $=1$ - mmap_write $=1$ - - cache_size $=100000$

-- cache_numshardbits $=0--$ sync $=0--$ verify_checksum $=0$

${ }^{15}$ https://www.kernel.org/doc/Documentation/locking/locktorture.txt
} 


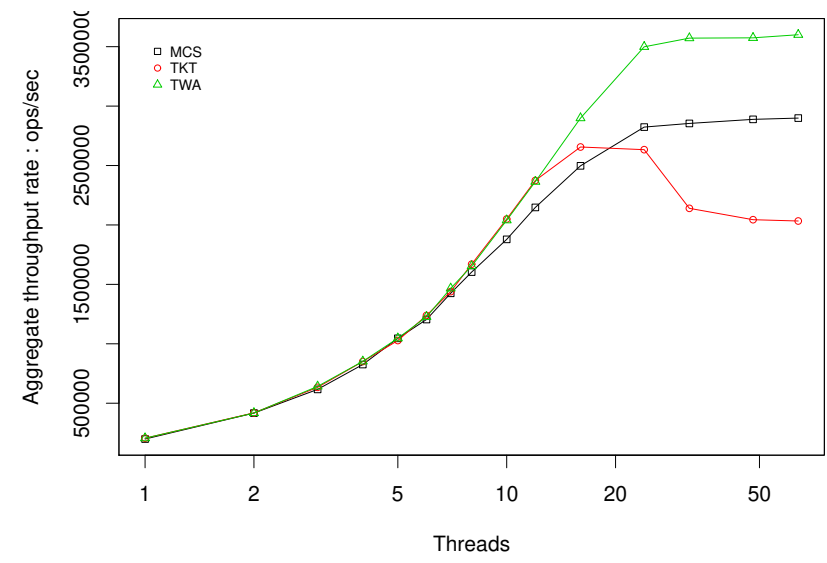

Figure 10. RocksDB readwhilewriting

and occasional long delays ("to force massive contention") inside the critical section. At the end of the measurement interval, it reports the overall throughput (lock acquisitions) completed by the threads. We used locktorture to compare TWA, classic ticket locks, and the default kernel qspinlock.

The Linux qspinlock construct [11, 12,31] is a compact 32-bit lock, even on 64-bit architectures. The low-order bits of the lock word constititue a simple test-and-set lock while the upper bits encode the tail of an MCS chain. The result is a hybrid of MCS and test-and-set ${ }^{16}$. In order to fit into a 32-bit work - a critical requirement - the chain is formed by logical CPU identifiers instead of traditional MCS queue node pointers. Arriving threads attempt to acquire the testand-set lock embedded in the low order bits of the lock word. This attempt fails if the test-and-set lock is held or of the MCS chain is populated. If successful, they enter the critical section, otherwise they join the MCS chain embedded in the upper bits of the lock word. When a thread becomes an owner of the MCS lock, it can wait for the test-and-set lock to become clear, at which point it claims the test-andset lock, releases the MCS lock, and then enters the critical section. The MCS aspect of qspinlock is used only when there is contention. The unlock operator simply clears the test-and-set lock. The MCS lock is never held over the critical section, but only during contended acquistion. Only the owner of the MCS lock spins on the test-and-set lock, reducing coherence traffic ${ }^{17}$. Qspinlock is strictly FIFO. While the technique employs local spinning on the MCS chain, unlike traditional MCS, arriving and departing threads will both update the common lock word, increasing coherence traffic and degrading performance relative to classic MCS. Qspinlock incorporates an additional optimization where the first

\footnotetext{
${ }^{16}$ https://github.com/torvalds/linux/blob/master/kernel/locking/qspinlock.

${ }^{17}$ This provides a LOITER-style [19] lock with the outer lock consisting of a test-and-set lock and the inner lock consisting of the MCS lock, with both locks embedded in the same 32-bit word.
}

contending thread spins on the test-and-set lock instead of using the MCS path. Traditional MCS does not fit well in the Linux kernel as (a) the contraint that a low-level spin lock instance be only 32-bits is a firm requirement, and (b) the lock-unlock API does not provide a convenient way to pass the owner's MCS queue node address from lock to unlock. We note that qspinlocks replaced classic ticket locks as the kernel's primary low-level spin lock mechanism in 2014, and ticket locks replaced test-and-set locks, which are unfair and allow unbounded bypass, in 2008 [13].

Regarding lock instance size, Bueso [10] notes:

Lock overhead. This is the resource cost of using a particular lock in terms of both size and latency. Locks embedded in data structures, for example, will bloat that type. Larger structure sizes mean more CPU cache and memory footprint. Thus, size is an important factor when a structure becomes frequently used throughout the system. Implementers also need to consider lock overhead when enlarging a lock type, after some nontrivial modification; this can lead to performance issues in unexpected places. For example, Linux kernel file-system and memory-management developers must take particular care of the size of VFS struct inode (index node) and struct page, optimizing as much as possible. These data structures represent, respectively, information about each file on the system and each of the physical page frames. As such, the more files or memory present, the more instances of these structures are handled by the kernel. It is not uncommon to see machines with tens of millions of cached inodes, so increasing the size of the inode by 4 percent is significant. That's enough to go from having a well-balanced workload to not being able to fit the working set of inodes in memory. Implementers must always keep in mind the size of the locking primitives.

Another example where the size of the lock is important is in concurrent data structures, such as linked lists or binary search trees, that use a lock per node or entry $[9,16,27]$. As Bronson at el. observe, when a scalable lock is striped across multiple cache lines to avoid contention in the coherence fabric, it is "prohibitively expensive to store a separate lock per node" [9].

In Table-1 we report the cyclomatic complexity [34] and $\mathrm{N}$-Path complexity $[7,36]$ measures - derived from the complexity of the control flow graph - for the lock and unlock methods for classic ticket locks (TKT), the kernel qspinlock primitive ${ }^{18}$ and TWA. To facilitate a fairer comparison, we removed performance monitoring and debugging facilities from qspinlock before running oclint ${ }^{19}$ to compute the complexity. In addition, the values reported above reflect

\footnotetext{
${ }^{18}$ https://github.com/torvalds/linux/blob/master/kernel/locking/qspinlock.

${ }^{19}$ https://github.com/oclint/oclint
} 
only the top-level qspinlock acquistion method, and does not include the complexity of helper methods that it calls. The only helper methods used by TWA and ticket locks are atomic operators and the PAUSE operator, which reduce to just one instruction with no additional complexity. All three lock algorithms have cyclomatic and N-Path complexity of just 1 for the unlock operation. As can be seen in the table, TWA's lock operator is far less complex than is the qspinlock acquisition method.

\begin{tabular}{lcclcc}
\hline & \multicolumn{2}{c}{ NPath } & & \multicolumn{2}{c}{ Cyclomatic } \\
\cline { 2 - 3 } \cline { 5 - 6 } & Lock & Unlock & & Lock & Unlock \\
\hline Ticket Lock & 2 & 1 & & 2 & 1 \\
QSpinLock & 4320 & 1 & & 18 & 1 \\
TWA & 28 & 1 & & 6 & 1 \\
\hline \hline
\end{tabular}

Table 1. Complexity Measures

The average critical section duration used by locktor ture is a function of the number of concurrent threads. In order to use the benchmark to measure and report scalability, we augmented it to parameterize the critical and noncritical section durations, which are expressed as steps of the thread-local pseudo-random number generator provided in the locktor ture infrastructure. We used 20 steps for the critical section. Each execution of the non-critical section computes a uniformly random distributed number in $[0-N]$ and then steps the local random number generator that many iterations. At the end of a run (lasting 30 seconds in our case), the total number of lock operations performed by all threads is reported. We report the median of 7 such runs. Figure-11 uses $N=20$ and Figure- 12 uses $N=400$.

For the kernel versions of ticket locks and TWA we reduced the ticket and grant fields to 16 bits to allow the composite lock word to fit in 32-bits, imposing a constraint that the maximum number of waiting threads never exceeds 65535. Qspinlocks operate with preemption disabled, so the constraint devolves to not having more than 65535 processors waiting on a given lock at any one time. By default, the maximum number of CPUs allowed by the Linux kernel on x86 architectures is 4096, satisfying the constraint.

As we can see in Figures 11 and 12, classic ticket locks perform well at low conconcurrency but fade as the number of threads increases. TWA and and QSpinLock provide fairly similar performance, although TWA is far simpler.

\section{Conclusion}

while our approach is deterministic we note that performance under TWA can be influenced by the activities of other unrelated threads and locks by means of collisions in the shared waiting array, potentially reducing predictability. Other shared resources incur the same risk. Examples

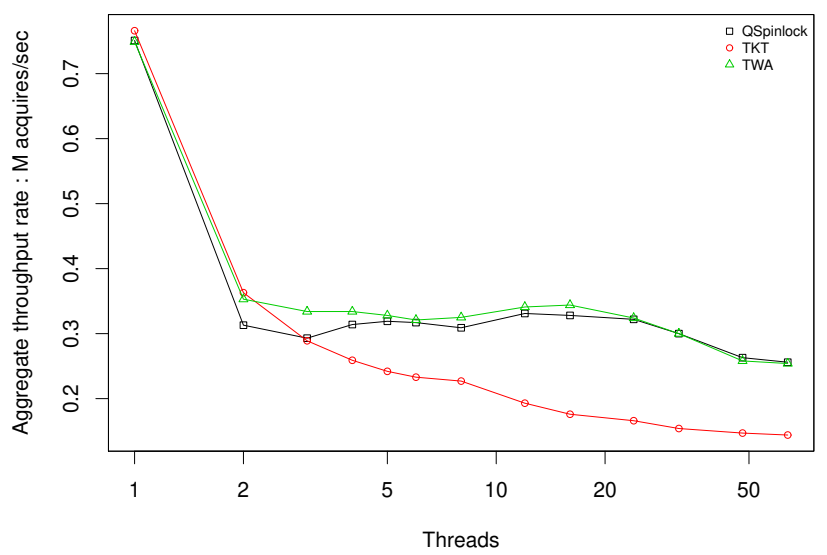

Figure 11. LockTorture : High Contention

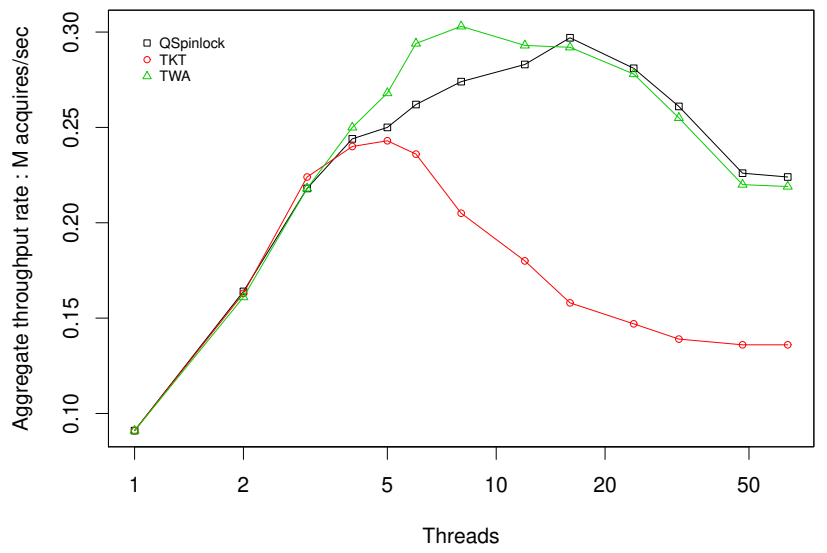

Figure 12. LockTorture : Moderate Contention

include (a) competition for occupancy of shared hardware caches and (b) collisions in the Linux futex hash table, where lock addresses map to hash chains of blocked threads.

TWA is a straightforward extension to classic ticket locks, providing the best performance properties of ticket locks and MCS locks. Like ticket locks, it is simple, compact, and has a fixed memory footprint. The key benefit conferred by TWA arises from improved transfer of ownership (handover) in the unlock path, by reducing the number of threads spinning on the grant field at any given time. Even though TWA increases the overall path length in the unlock operation, adding an atomic fetch-and-increment operation compared to the classic ticket lock, it decreases the effective critical path duration for contended handover.

We plan to explore long-term waiting in the Linux kernel via the MONITOR-MWAIT construct or by means of the kernel futex [23] mechanism.

An extended version of this paper is available at https://arxiv.org/abs/1810. 01573 


\section{Acknowledgments}

We thank Shady Issa for reviewing drafts and helpful comments.

\section{References}

[1] 2016. RFC : introduce dl_iterate_phdr_parallel. https://patchwork. ozlabs.org/patch/652301/

[2] Yehuda Afek, Dave Dice, and Adam Morrison. 2011. Cache Index-aware Memory Allocation. In Proceedings of the International Symposium on Memory Management (ISMM '11). ACM. http://doi.acm.org/10.1145/ 1993478.1993486

[3] Vitaly Aksenov, Dan Alistarh, and Petr Kuznetsov. 2018. Brief Announcement: Performance Prediction for Coarse-Grained Locking. In Proceedings of the 2018 ACM Symposium on Principles of Distributed Computing (PODC '18). ACM. https://doi.org/10.1145/3212734.3212785

[4] J.H. Anderson, Y.J. Kim, and T. Herman. 2003. Shared-memory mutual exclusion: major research trends since 1986. Distributed Computing (2003). https://doi.org/10.1007/s00446-003-0088-6

[5] T. E. Anderson. 1990. The performance of spin lock alternatives for shared-money multiprocessors. IEEE Transactions on Parallel and Distributed Systems (1990). https://doi.org/10.1109/71.80120

[6] Jelena Antić, Georgios Chatzopoulos, Rachid Guerraoui, and Vasileios Trigonakis. 2016. Locking Made Easy. In Proceedings of the 17th International Middleware Conference (Middleware '16). ACM. https: //doi.org/10.1145/2988336.2988357

[7] Lucas Bang, Abdulbaki Aydin, and Tevfik Bultan. 2015. Automatically Computing Path Complexity of Programs. In Proceedings of the 2015 10th foint Meeting on Foundations of Software Engineering (ESEC/FSE 2015). ACM. http://doi.acm.org/10.1145/2786805.2786863

[8] Silas Boyd-Wickizer, M. Frans Kaashoek, Robert Morris, and Nickolai Zeldovich. 2012. Non-scalable locks are dangerous. Ottawa Linux Symposium (OLS) (2012). https://www.kernel.org/doc/ols/2012/ ols2012-zeldovich.pdf

[9] Nathan G. Bronson, Jared Casper, Hassan Chafi, and Kunle Olukotun. 2010. A Practical Concurrent Binary Search Tree. In Proceedings of the 15th ACM SIGPLAN Symposium on Principles and Practice of Parallel Programming (PPoPP '10). ACM. http://doi.acm.org/10.1145/1693453. 1693488

[10] Davidlohr Bueso. 2014. Scalability Techniques for Practical Synchronization Primitives. Commun. ACM (2014). http://doi.acm.org/10 $1145 / 2687882$

[11] Jonathan Corbet. [n. d.]. Cramming more into struct page. https: //lwn.net/Articles/565097, August 28, 2013. Accessed: 2018-10-01.

[12] Jonathan Corbet. [n. d.]. MCS locks and qspinlocks. https://wn.net/ Articles/590243, March 11, 2014. Accessed: 2018-09-12.

[13] Jonathan Corbet. [n. d.]. Ticket Spinlocks. https://lwn.net/Articles/ 267968, February 6, 2008. Accessed: 2018-09-12.

[14] Jonathan Corbet. 2014. MCS locks and qspinlocks. https://lwn.net/ Articles/590243/

[15] Oracle Corporation. 2014. SPARC T7-2 Server - Oracle Datasheet. http:/www.oracle.com/us/products/servers-storage/ sparc-t7-2-server-ds-2687049.pdf

[16] Tyler Crain, Vincent Gramoli, and Michel Raynal. 2012. A Speculationfriendly Binary Search Tree. In Proceedings of the 17th ACM SIGPLAN Symposium on Principles and Practice of Parallel Programming (PPoPP '12). ACM. http://doi.acm.org/10.1145/2145816.2145837

[17] Tudor David, Rachid Guerraoui, and Vasileios Trigonakis. 2013. Everything You Always Wanted to Know About Synchronization but Were Afraid to Ask. In Proceedings of the Twenty-Fourth ACM Symposium on Operating Systems Principles (SOSP '13). http://doi.acm.org/10.1145/ 2517349.2522714

[18] David Dice. 2011. Brief Announcement: A Partitioned Ticket Lock. In Proceedings of the Twenty-third Annual ACM Symposium on Parallelism in Algorithms and Architectures (SPAA '11). http://doi.acm.org/10. 1145/1989493.1989543

[19] Dave Dice. 2017. Malthusian Locks. In Proceedings of the Twelfth European Conference on Computer Systems (EuroSys '17). http://doi. acm.org/10.1145/3064176.3064203

[20] David Dice, Virendra J. Marathe, and Nir Shavit. 2015. Lock Cohorting: A General Technique for Designing NUMA Locks. ACM Trans. Parallel Comput. (2015). https://doi.org/10.1145/2686884

[21] Stijn Eyerman and Lieven Eeckhout. 2010. Modeling Critical Sections in Amdahl's Law and Its Implications for Multicore Design. In Proceedings of the 37th Annual International Symposium on Computer Architecture (ISCA '10). ACM. https://doi.org/10.1145/1815961.1816011

[22] M. J. Fischer, N. A. Lynch, J. E. Burns, and A. Borodin. 1979. Resource allocation with immunity to limited process failure. In 20th Annual Symposium on Foundations of Computer Science (FOCS 1979). http: //dx.doi.org/10.1109/SFCS.1979.37

[23] Hubertus Franke, Rusty Russel, and Matthew Kirkwood. [n. d.]. Fuss, Futexes and Furwocks: Fast User-level Locking in Linux. https://www. kernel.org/doc/ols/2002/ols2002-pages-479-495.pdf. Ottawa Linux Symposium.

[24] S. S. Fu and Nian-Feng Tzeng. 1997. A circular list based mutual exclusion scheme for large shared-memory multiprocessors. IEEE Transactions on Parallel and Distributed Systems (1997). https://doi. org/10.1109/71.595581

[25] J.R. Goodman and H.H.J. Hum. 2009. MESIF: A Two-Hop Cache Coherency Protocol for Point-to-Point Interconnects. https://www.cs. auckland.ac.nz/ goodman/TechnicalReports/MESIF-2009.pdf

[26] P. H. Ha, M. Papatriantafilou, and P. Tsigas. 2005. Reactive spin-locks: a self-tuning approach. In 8th International Symposium on Parallel Architectures,Algorithms and Networks (ISPAN'05). https://doi.org/10. 1109/ISPAN.2005.73

[27] Steve Heller, Maurice Herlihy, Victor Luchangco, Mark Moir, William N. Scherer, and Nir Shavit. 2006. A Lazy Concurrent Listbased Set Algorithm. In Proceedings of the 9th International Conference on Principles of Distributed Systems (OPODIS'05). Springer-Verlag. http://dx.doi.org/10.1007/11795490_3

[28] Maurice Herlihy and Nir Shavit. 2008. The Art of Multiprocessor Programming. Morgan Kaufmann Publishers Inc.

[29] Ting-Lu Huang and Chien-Hua Shann. 1998. A Comment on "A Circular List-Based Mutual Exclusion Scheme for Large Shared-Memory Multiprocessors". IEEE Transactions on Parallel and Distributed Systems (1998). http://dx.doi.org/10.1109/71.667901

[30] Beng-Hong Lim and Anant Agarwal. 1994. Reactive Synchronization Algorithms for Multiprocessors. In Proceedings of the Sixth International Conference on Architectural Support for Programming Languages and Operating Systems (ASPLOS VI). ACM. https://doi.org/10.1145/ 195473.195490

[31] Waiman Long. [n. d.]. qspinlock: Introducing a 4-byte queue spinlock implementation. https://lwn.net/Articles/561775, July 31, 2013. Accessed: 2018-09-19.

[32] O. Krieger B. Rosenburg M. Auslander, D. Edelsohn and R. Wisniewski. 2003. Enhancement to the MCS lock for increased functionality and improved programmability - U.S. patent application number 20030200457. https://patents.google.com/patent/US20030200457

[33] George Marsaglia. 2003. Xorshift RNGs. Journal of Statistical Software, Articles (2003). https://doi.org/10.18637/jss.v008.i14

[34] T. J. McCabe. 1976. A Complexity Measure. IEEE Transactions on Software Engineering (1976). https://doi.org/10.1109/TSE.1976.233837

[35] John M. Mellor-Crummey and Michael L. Scott. 1991. Algorithms for Scalable Synchronization on Shared-memory Multiprocessors. ACM Trans. Comput. Syst. (1991). http://doi.acm.org/10.1145/103727.103729

[36] Brian A. Nejmeh. 1988. NPATH: A Measure of Execution Path Complexity and Its Applications. Commun. ACM (1988). http: //doi.acm.org/10.1145/42372.42379 
[37] Pedro Ramalhete. 2015. Ticket Lock - Array of Waiting Nodes (AWN). http://concurrencyfreaks.blogspot.com/2015/01/ ticket-lock-array-of-waiting-nodes-awn.html

[38] Pedro Ramalhete and Andreia Correia. 2016. Tidex: A Mutual Exclusion Lock. In Proceedings of the 21st ACM SIGPLAN Symposium on Principles and Practice of Parallel Programming (PPoPP '16). http://doi.acm.org/ $10.1145 / 2851141.2851171$

[39] David P. Reed and Rajendra K. Kanodia. 1979. Synchronization with Eventcounts and Sequencers. Commun. ACM (1979). http://doi.acm. org $/ 10.1145 / 359060.359076$

[40] Michael L. Scott. 2013. Shared-Memory Synchronization. Morgan \& Claypool Publishers.

[41] Peter Sewell, Susmit Sarkar, Scott Owens, Francesco Zappa Nardelli, and Magnus O. Myreen. 2010. X86-TSO: A Rigorous and Usable Programmer's Model for x86 Multiprocessors. Commun. ACM (2010). http://doi.acm.org/10.1145/1785414.1785443

[42] U. Verner, A. Mendelson, and A. Schuster. 2017. Extending Amdahl's Law for Multicores with Turbo Boost. IEEE Computer Architecture Letters (2017). https://doi.org/10.1109/LCA.2015.2512982

[43] Wikipedia contributors. 2018. Birthday problem - Wikipedia, The Free Encyclopedia. https://en.wikipedia.org/w/index.php?title=Birthday problem\&oldid=853622452. [Online; accessed 8-August-2018].

\section{Appendix : Algorithmic Variations}

- TKT-Dual An interesting variation on TWA is to forgo the waiting array and simply augment the ticket lock structure to use two grant fields, one for short-term waiting, for the immediate successor and perhaps a small number of other threads "near" the front of conceptual queue, and a second grant field for long-term waiting. To reduce coherence traffic we isolated the grant field to be sole occupant of a cache sector, increasing the lock size. The unlock operator first advances the short-term grant field, and, as needed, may advance the long-term grant field to shift one or more threads from long-term to short-term waiting, constraining the number of short-term waiters and accelerating handover. We refer to this form as TKT-Dual given the dual encoding of the grant field. Initial experiments with this form show promise, yielding results better than that of the baseline ticket lock, although lagging slightly behind TWA. The increased size means this form can not be used in the linux kernel as a replacement for the qspinlock algorithm.

- TWA-Staged We are also exploring variations of TWA where the fast uncontended path for both lock and unlock operations would be identical to that of normal ticket locks, specifically avoiding accesses to the waiting array in the unlock operator. Briefly, we divide waiting threads into 3 groups: (A) Those that are 2 or more elements away from the front of the conceptual queue. These "long-term" threads wait via the waiting array in the usual TWA fashion. (B) The thread that is 2 away from the head. This "transitional" thread busy-waits on the global grant field. (C) The thread that is 1 away from the head. This is the immediate successor, and also busy-waits on the global grant field. Assume we have a non-trivial set of waiting threads. Incrementing grant in the unlock operator passes ownership to the thread $T 1$ in (C) state above. T1 exits (C) state and becomes the owner. Thread T2 in (B) state also observes the change in the grant field, at which point it increments the waiting array slot associated with the next ticket value - the ticket value one after it's assigned ticket - to transfer a thread from (A) state to take $T 2$ 's place as the (B) thread. T2 then shifts from (B) to $(\mathrm{C})$ state. The downside to this approach is that we have 2 threads directly busy-waiting on grant, the (B) and (C) threads, whereas in our other TWA variants we at most have one thread busy-waiting on grant. The upside is that unlock operator does not access the waiting array, and all the waiting array accesses - both loads and fetch-and-add operations - are performed by waiting threads. As noted above, the unlock operator simply increments the grant field. This approach leverages those waiting threads to help drive through the $(\mathrm{A}) \rightarrow(\mathrm{B}) \rightarrow(\mathrm{C})$ transitions, reducing the path length of the unlock operation. Relative to classic ticket locks, all additional code is encapsulate in the locking slow path. In the case of uncontended operations, the array is never accessed and the path is the same as classic ticket locks, improving uncontended unlock latency.

- TWA-ID We note that we can replace the atomic increment of the waiting array element in the unlock path with a simple store of 0 to that location by changing the waiting array elements from counters to unique thread identity references. Threads arriving in the long-term waiting state will write their temporally unique non-zero identity value (which can be as simple as the address of the stack pointer) into the waiting array, recheck the grant value and then busy-wait while the waiting array elements remains equal to the thread identity value they just stored. When the element changes, the thread shifts to classic short-term waiting. While this approach eliminates the atomic fetch-and-add in the unlock path, it also increases write traffic into the shared array, as threads entering the long-term write phase must store their unique identity.

- TWA-Quantized To reduce the impact on the unlock path we can arrange for the unlock operator to release a quanta or gang of $N$ threads - based on the ticket lock values - into global spinning mode every Nth unlock operation. This reduces the average cost of an unlock operation, but increases the number of threads waiting via global spinning.

- 3-Stage A key observation in the design of TWA is importance of hand-over efficiency, improved by reducing the number of threads concurrently busy-waiting on the grant field. This insight informs the design of other locks that perform gracefully under contention. One such variation is a 3-Stage mutex $M$ which has 3 sub-locks : $A, B$ and $C$. $A$ would typically by a FIFO lock such as MCS or ticket $B$ and $C$ could be simple test-and-set locks. To acquire $M$ a thread uses the following protocol : acquire $A$; acquire $B$; release $A$; acquire $C$; release $B$. To release $M$ a thread simply releases $C$. The 
thread that holds $C$ also holds $M$ itself. (For efficiency, we might shift the release of $B$ to unlock path).

This approach, while seemingly complicated, confers a number of advantages. If we have $N$ waiting threads, then $N-2$ will typically wait on $A, 1$ on $B$ and 1 on $C$. When we release $M$, by releasing $C$, there is at most one thread waiting on $C$, so handover costs arising from coherence traffic are minimized. Once a thread has acquired $C$ it still needs to release $B$, and this occurs within the critical section of $M$. But there is at most one waiter on $B$, so the cost to release $B$ isn't excessive. A relatively large handover cost may be incurred when we release $A$, but that latency overlaps with execution of the critical section and does not manifest as reduced throughput. The latency associated with the release of $A$ is subsumed into the waiting period for $M$ and moved out of the critical path, improving throughput and scalability. Note that $M$ is FIFO if $A$ is FIFO. A protects the acquisition of $B$, so as an optional optimization we can avoid atomics to acquire $B$. And $B$ likewise protects the acquisition of $C$.

To reduce the path length in the uncontended case, we can add a fast-path where arriving threads trylock directly on $C$, and, if successful can avoid $A$ and $B$. In this case, however, we would need to use atomics to acquire $C$ in the slow path.

Finally, we believe that replacing the waiting array elements with pointers to chains of waiting threads may have benefit. Briefly, each long-term waiting thread would have an on-stack MCS-like queue node that it would push into the appropriate chain in the waiting array, and then use local spinning on a field within that node. Notification of longterm waiters causes the chain to be detached via an atomic SWAP instruction and all the elements are updated to reflect that they should reevaluate the grant field. In the case of collisions, waiting threads may need to re-enqueue on the chain. This design recapitulates much of the Linux kernel "futex" mechanism.

\section{Appendix: Maximum Ideal Scalability}

We note that our system-under-test does not exhibit ideal linear scalability as we increase the number of threads. To demonstrate this effect we use a IdealScalabilty microbenchmark which spaws $T$ concurrent threads, each of which loops advancing a thread-local std: : mt 19933 pseudo-random number generator. There is no sharing, no communication, and no waiting; the threads are completely independent. At the end of a 30 second measurement interval the benchmark tallies and reports the aggregate number of random number steps completed. We report the median of 5 such runs for each data point. In Figure 13 we vary $T$ on the $\mathrm{X}$-axis, and on the Y-axis we report the normalized throughput throughput divided by the throughtput at $T=1-$ divided by the number of participating threads $T$. This yields a fraction which reflects the slow-down caused by fraternal interference for shared hardware compute resources, such as caches, cores, pipelines, DRAM channels, etc. Specifically, we observe that the progress rate of each thread is impeded by the concurrent execution of unrelated threads.

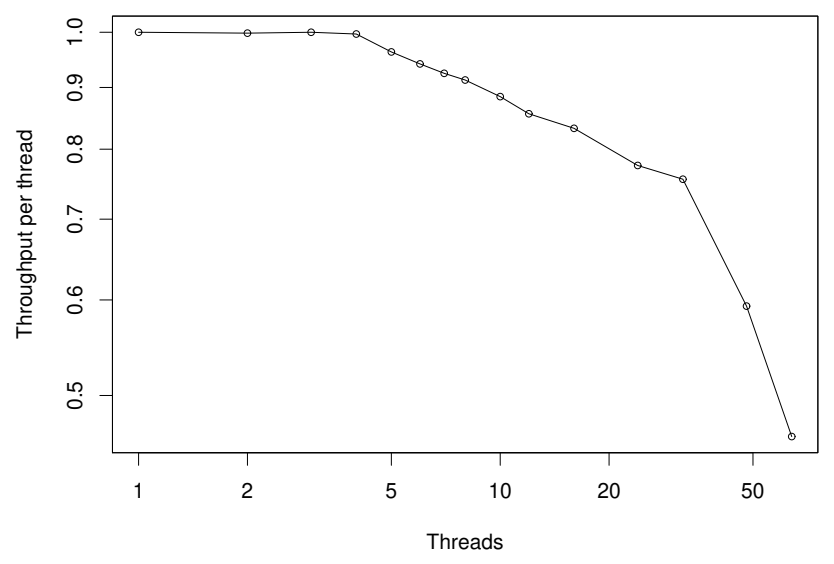

Figure 13. Maximum Ideal Scalability 